\title{
Folic Acid-Conjugated Cellulose Nanocrystals Show High Folate- Receptor Binding Affinity and Uptake by KB and Breast Cancer Cells
}

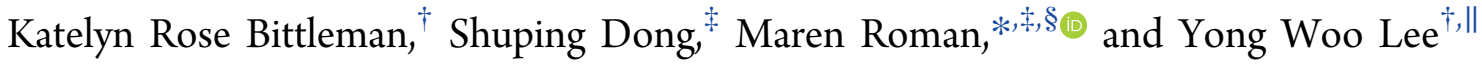 \\ ${ }^{\dagger}$ Virginia Tech-Wake Forest University School of Biomedical Engineering and Sciences (MC0298), 325 Stanger Street, Blacksburg, \\ Virginia 24061, United States \\ ${ }^{\ddagger}$ Macromolecules Innovation Institute (MC0201), Virginia Tech, 1075 Life Science Circle, Blacksburg, Virginia 24061, United \\ States \\ ${ }^{\S}$ Department of Sustainable Biomaterials (MC0323), Virginia Tech, 310 West Campus Drive, Blacksburg, Virginia 24061, United \\ States \\ "Department of Biomedical Engineering and Mechanics (MC0298), Virginia Tech, 325 Stanger Street, Blacksburg, Virginia 24061, \\ United States
}

\section{Supporting Information}

\begin{abstract}
The study evaluates cellulose nanocrystals (CNCs) as nanocarriers for targeted, intracellular delivery of molecular agents. CNCs were labeled with fluorescein-5'isothiocyanate as an imaging agent and conjugated to folic acid (FA) as a targeting ligand. The $\mathrm{CNC}$ conjugates were characterized by UV-vis spectroscopy, $\zeta$-potential analysis, dynamic light scattering, and atomic force microscopy. Cellular binding/uptake of the FA-conjugated CNCs by $\mathrm{KB}$ and MDA-MB-468 cells was quantified with cellular uptake assays. Internalization of the particles was confirmed by confocal microscopy. Uptake mechanisms were determined by inhibition studies with chlorpromazine and genistein. Binding

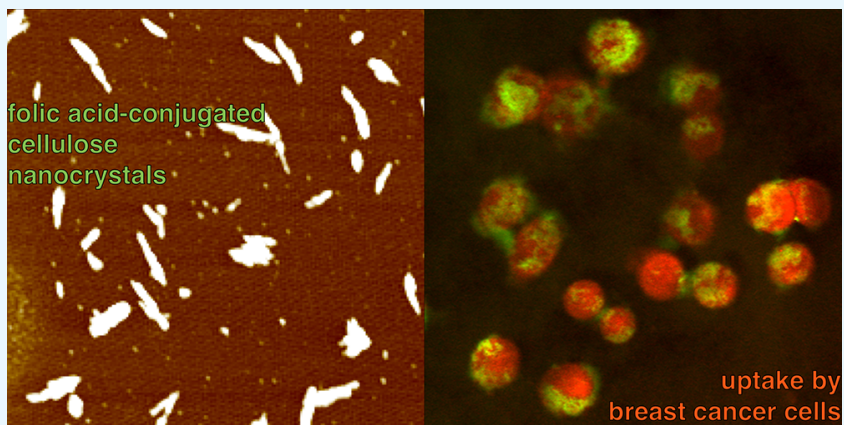
affinity was qualitatively assessed with a free folate inhibition assay. Both KB and MDA-MB-468 cells exhibited significant and folate-receptor specific binding/uptake of FA-conjugated CNCs. Clathrin-mediated endocytosis was a significant uptake mechanism in both cell types, whereas caveolae-mediated endocytosis only played a significant role in MDA-MB-468 cells. Uptake inhibition of FA-conjugated CNCs by $\mathrm{KB}$ cells required high concentrations $(>1 \mathrm{mM})$ of free FA. The observed FRspecific internalization of FA-conjugated CNCs by FR-positive cancer cells and tumors and their remarkable high affinity for the FR demonstrate the great potential of $\mathrm{CNCs}$ as novel nanocarriers for imaging agents and chemotherapeutics in the early detection and treatment of cancer.
\end{abstract}

\section{INTRODUCTION}

One of the most promising applications of nanotechnology is the selective delivery of molecules to specific cells of the body. This can be accomplished with novel nanoscale delivery systems in which selective targeting agents, therapeutic drugs, and/or imaging probes are conjugated to the surface or encapsulated within the particle. ${ }^{1-4}$ As one of several targeting strategies, receptor-mediated cell targeting has become an attractive approach for the early detection and treatment of cancer in recent years, and many suitable cancer-specific receptors have been identified. ${ }^{5}$ Many factors need to be considered in the design of nanoscale carrier systems for imaging or therapeutic applications. One must optimize parameters, such as particle shape, size, surface chemistry, cytotoxicity, and circulation time. ${ }^{6}$ Decuzzi et al. investigated optimal size and shape of nanoparticles for increased circulation time and accumulation at tumor sites. Spherical particles were shown to be inefficient because they tend to flow toward the center of blood vessels with laminar flow, whereas irregularly shaped or high-aspect-ratio nanoparticles tend to be pushed to the walls of blood vessels, similar to platelets. This increases the likelihood of nanoparticles entering tumor tissues through fenestrations in the vasculature. ${ }^{6,7}$

Cellulose nanocrystals (CNCs) are elongated nanoparticles ranging in average length from 100 to $200 \mathrm{~nm}$ and average height from 3 to $5 \mathrm{~nm}$ if derived from wood pulp. ${ }^{8}$ This size range is expected to be too large for rapid renal clearance yet small enough for evasion of the mononuclear phagocytic system. ${ }^{9}$ However, because of their elongated shape, CNCs can be expected to orient themselves in the direction of blood flow and eventually pass through glomerular fenestrations in the kidney, enabling ultimate excretion in the urine. ${ }^{10}$ Further-

Received: July 11, 2018

Accepted: October 11, 2018

Published: October 24, 2018 
Table 1. Properties of CNCs and CNC Conjugates

\begin{tabular}{|c|c|c|c|c|c|c|c|c|c|}
\hline \multirow[b]{2}{*}{ sample } & \multicolumn{2}{|c|}{ cellulose content } & \multicolumn{2}{|c|}{ FITC content } & \multicolumn{2}{|c|}{ FA content } & \multicolumn{2}{|c|}{ hydrodynamic diameter } & \multirow[b]{2}{*}{$\zeta$-potential $(\mathrm{mV})^{b}$} \\
\hline & (wt \%) & $(\mathrm{mol} \%)^{a}$ & (wt \%) & $(\mathrm{mol} \mathrm{\%})$ & (wt \%) & $(\mathrm{mol} \%)$ & $(\mathrm{nm})^{b}$ & $(\mathrm{PDI})^{c}$ & \\
\hline CNCs & 100.0 & 100.0 & 0 & 0 & 0 & 0 & $81 \pm 2$ & 0.23 & $-47 \pm 2$ \\
\hline FITC-CNC & 92.6 & 96.8 & 7.4 & 3.2 & 0 & 0 & $109 \pm 23$ & 0.36 & $-37 \pm 8$ \\
\hline FITC-CNC-FA & 73.4 & 88.1 & 3.7 & 1.8 & 22.9 & 10.1 & $204 \pm 50$ & 0.24 & $-40 \pm 4$ \\
\hline
\end{tabular}

${ }^{a}$ On the basis of the molar mass of the anhydroglucose unit. ${ }^{b}$ Data shown are means \pm standard deviations. ${ }^{c}$ PDI: polydispersity index.

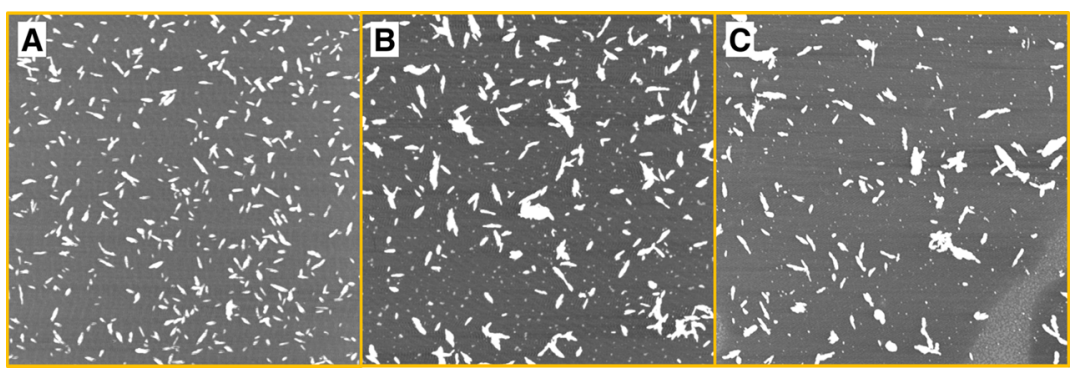

Figure 1. AFM images of CNCs (A), FITC-CNC (B), and FITC-CNC-FA (C). Images were recorded in intermittent contact mode under ambient conditions with tips of less than $10 \mathrm{~nm}$ nominal tip radius. Image size is $5 \mu \mathrm{m} \times 5 \mu \mathrm{m}$ and the z-scale (black to white) is $6 \mathrm{~nm}$.

more, their high aspect ratio likely causes increased fenestration penetration in tumor vasculature compared to spherical particles, which many carriers are by design, ${ }^{11}$ e.g., liposomes, ${ }^{12-14}$ metal nanoparticles, ${ }^{15,16}$ and dendrimers. ${ }^{17,18}$ The starting material, cellulose, is extremely abundant in nature, inexpensive, and has excellent strength properties. ${ }^{4,19,20}$ Additionally, the surface of CNCs contains multiple hydroxyl groups, which are amenable to chemical modification for ligand targeting, labeling with imaging probes, and drug conjugating. Finally, toxicity studies have indicated a lack of or low toxicity for CNCs. ${ }^{21}$

Folate receptors (FRs), mediating cellular uptake of folic acid (FA), also known as vitamin $\mathrm{B}_{9}$, are overexpressed on the plasma membrane of many cancer cell types, including breast, ovarian, lung, kidney, brain, and endometrial cancer, whereas normal tissues seldom express the FRs. ${ }^{22-27}$ FA is commonly used as targeting agent for the FR because of its high affinity. FA is a key vitamin for cell division and is needed by all cells to proliferate. However, healthy cells can also take up other forms of folate via the reduced folate carrier and the proton-coupled folate transporter, which does not actively bind FA. ${ }^{28,29}$ Overexpression of the FR is essential for rapidly dividing cells, such as cancer cells.

Development of a noninvasive, inert screening strategy would greatly reduce late-stage diagnoses of cancer, minimize unnecessary risk to patients, and increase chances of survival. According to the American Cancer Society, the 5 year survival rate for lung cancer increases from 4 to $54 \%$ when diagnosed in early versus late stage. Likewise, kidney cancer has a 5 year survival rate of 92 versus $12 \%$ with early- and late-stage diagnoses, respectively. ${ }^{30}$ Currently, cancer screening methods are limited to breast, ovarian, colorectal, and prostate cancers. ${ }^{31}$ Since the FR is overexpressed in many cancer types, targeting strategies for the FR might enable us to screen for additional cancers, such as lung, kidney, and brain cancers, whose early detection would be extremely useful.

In a previous study, we showed that fluorescently labeled, FA-conjugated CNCs enable the detection of FR-positive human (DBTRG-05MG, H4) and rat (C6) brain tumor cells. $^{32}$ In the study reported here, we investigated uptake of these particles by KB and human breast cancer cells (MDA-
MB-468). We present confocal microscopy images that unequivocally and for the first time demonstrate internalization of FA-conjugated CNCs by FR-positive cancer cells. Furthermore, with a competition assay we show that FAconjugated $\mathrm{CNCs}$ have a remarkably high affinity for the FR.

\section{RESULTS AND DISCUSSION}

CNCs were synthesized from dissolving-grade, bleached wood pulp and functionalized with fluorescein- 5 -isothiocyanate (FITC) for detection and quantification. The fluorescently labeled CNCs (FITC-CNC) were subsequently functionalized with FA for FR targeting. Measurements of the FITC and FA contents by UV-vis spectroscopy yielded FITC contents of 7.4 and 3.7 wt \% for FITC-CNC and the FA-conjugate (FITCCNC-FA), respectively, and an FA content of 22.9 wt \% for FITC-CNC-FA (Table 1). $\zeta$-Potential analysis showed that both FITC-CNC and FITC-CNC-FA had a negative particle surface charge that was similar to that of unmodified CNCs. Particle analysis by dynamic light scattering (DLS) revealed that the hydrodynamic diameters of FITC-CNC and FITCCNC-FA were larger than that of unmodified CNCs. However, atomic force microscopy (AFM) images confirmed that both conjugates still had the initial elongated morphology (Figure 1).

FR- $\alpha$ expression by $\mathrm{KB}$ and MDA-MB-468 cells was quantified by real-time reverse transcription-polymerase chain reaction (RT-PCR) and immunofluorescent staining. KB and MDA-MB-468 cells had significantly higher levels of FR- $\alpha$ mRNA and protein expression than human aortic endothelial cells (HAEC), the negative control (Figure S1). FR- $\alpha$ mRNA expression of $\mathrm{KB}$ cells was over 8 million fold higher and that of MDA-MB-468 cells over 7000-fold higher than FR- $\alpha$ mRNA expression of HAEC (Figure S1a). Little to no expression of FR- $\alpha$ protein was detected in HAEC, however, KB and MDAMB-468 cells had detectable protein expression levels for FR- $\alpha$ (Figure S1b).

Incubation of $\mathrm{KB}$ and MDA-MB-468 cells in the presence of free FITC or nontargeted FITC-CNC resulted in little to no binding/uptake of these entities (Figure 2); furthermore, binding/uptake of FITC-CNC was lower than that of free 

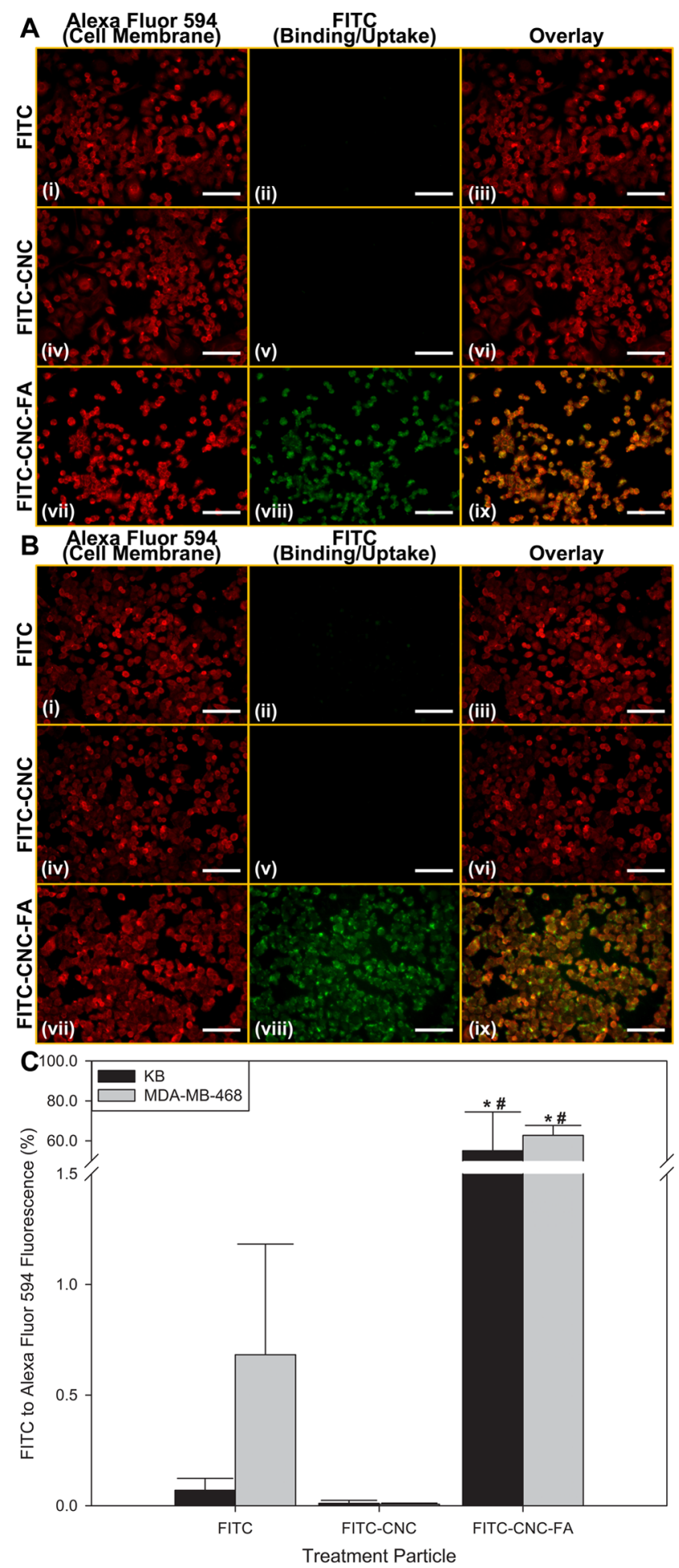

Figure 2. Cellular binding/uptake of free FITC (i-iii), FITC-CNC (iv-vi), and FITC-CNC-FA (vii-ix). KB (A) and MDA-MB-468 (B) cells were exposed to either free FITC or FITC-CNC conjugates for 2 h, stained with Alexa Fluor 594, and imaged by fluorescence microscopy. Images shown in (A) and (B) are representative of cell membrane (i, iv, vii), bound/uptaken FITC (ii, v, viii), and overlay images (iii, vi, ix) for each group $(n=4)$ (bar: $100 \mu \mathrm{m})$. Quantitative analysis of bound/uptaken particles (C). Data shown are means \pm SDs for each group $(n=4)$ of relative fluorescence intensity normalized against cell membrane area $\left({ }^{*} p<0.05\right.$ vs free FITC, ${ }^{\#} p<$ 0.05 vs FITC-CNC).
FITC. FITC molecules $\left(\mathrm{pK}_{\mathrm{a}}=4.4\right)$ are known to enter cells in their neutral form by passive diffusion across the plasma membrane, ${ }^{33}$ which may explain the greater uptake of FITC molecules relative to that of the larger, more hydrophilic, and negatively charged FITC-CNC particles. Incubation in the presence of FR-targeted FITC-CNC-FA resulted in significant binding/uptake of the nanoparticles (Figure 2), many times greater (>1000 times that of FITC-CNC) than could be expected from the slightly higher CNC concentration (99.2 $\mu \mathrm{g} / \mathrm{mL}$ for FITC-CNC-FA versus $62.6 \mu \mathrm{g} / \mathrm{mL}$ for FITC-CNC, particle concentrations were normalized by FITC content). The fact that FITC-CNC-FA is bound/internalized despite its negative charge indicates that charge-charge repulsion is sufficiently suppressed at the ionic strength of the cell culture medium or weaker than the receptor-ligand affinity.

To confirm targeting specificity, we conducted a competition assay in which $\mathrm{KB}$ cells were incubated in the presence of FITC-CNC-FA and increasing amounts of free FA. As the free FA concentration increased to $25 \mathrm{mM}$, decreasing amounts of FITC-CNC-FA were bound/taken up by KB cells (Figure 3 ). The decrease in binding/uptake of FITC-CNC-FA in the presence of free FA suggests that FITC-CNC-FA uptake by FR-negative cells will be minimal, i.e., that FITC-CNC-FA is selective toward FR-positive cells. The high concentration of free FA that was needed to significantly reduce FITC-CNC-FA binding/uptake by $\mathrm{KB}$ cells is remarkable. Previous studies showed a significant decrease in binding/uptake of FA conjugates at free FA concentrations of $1 \mathrm{mM}$ or less. ${ }^{34-38}$ Here, although the concentration of bound FA in the culture medium was only $0.07 \mathrm{mM}$, a free FA concentration of $5 \mathrm{mM}$ caused a smaller than $40 \%$ reduction in FITC-CNC-FA binding/uptake. Nukolova et al. ${ }^{39}$ reported a similar observation for folate-decorated nanogels. It has been suggested that the carboxyl group ( $\alpha$ or $\gamma$ ) used to conjugate folate to other ligands or particles has a direct effect on the affinity of FA for the FR. Leamon et $\mathrm{al}^{40}$ have further suggested that FR-mediated endocytosis is strongly influenced by the steric environment around the folate's 4-aminobenzoic acid moiety. The apparently high binding affinity of FITCCNC-FA suggests that low concentrations are sufficient to achieve significant cellular uptake in vivo at human serum folate concentrations $(9-14 \mathrm{nM}))^{35}$ Preliminary targeting experiments in a mouse model (Figure S2) indicated in vivo targeting efficacy.

Next, we investigated the mechanism for endocytosis of the FR-targeted CNC conjugates. The endocytotic pathway via FR is known to be mediated by two proteins, clathrin and caveolin. $^{41} \mathrm{~KB}$ and MDA-MB-468 cells pretreated with a clathrin inhibitor showed significantly reduced binding/uptake of FITC-CNC-FA (Figure 4). In MDA-MB-468 cells, binding/ uptake of FITC-CNC-FA was also significantly reduced by a caveolae inhibitor. These results suggest that the mechanism for FITC-CNC-FA uptake via the FR is cell-dependent and nonexclusive. Both cell types exhibited primarily clathrinmediated endocytosis. However, in MDA-MB-468 cells, caveolae-mediated endocytosis was also significant. Many studies have reported cell type-specific dependence on either clathrin or caveolin for FR-mediated endocytosis. ${ }^{42-44}$ In our previous study, human and rat astrocytoma cells (DBTRG05MG and C6, respectively), which are non-neuronal brain cells, internalized FITC-CNC-FA primarily via caveolaemediated endocytosis, whereas human ganglioma cells (H4), which are neuronal brain cells, internalized FITC-CNC-FA 

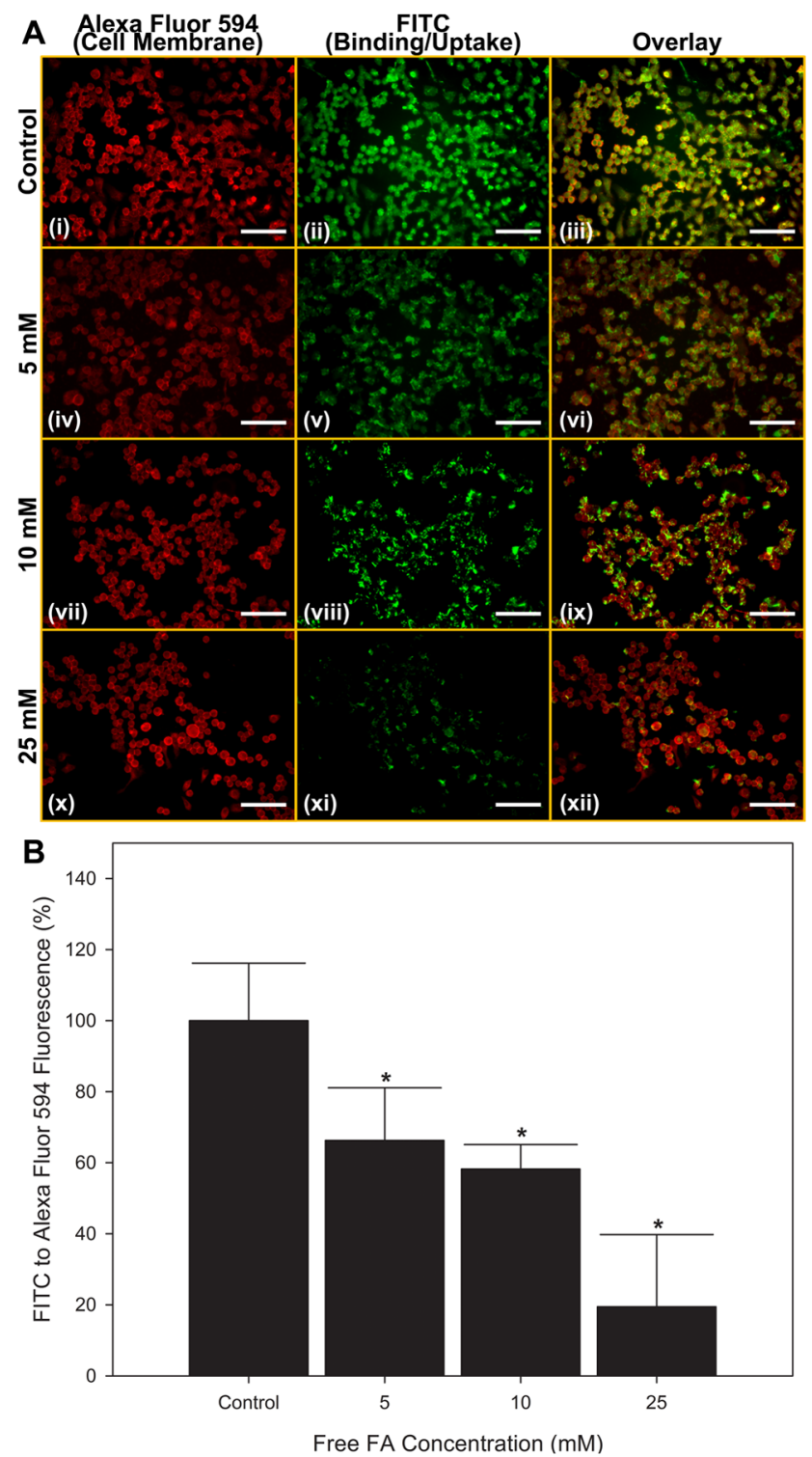

Figure 3. Effects of free FA on cellular binding/uptake of FITCCNC-FA by KB cells. Cells were exposed to FITC-CNC-FA and increasing concentrations of FA for 2 h, stained with Alexa Fluor 594, and imaged by fluorescence microscopy. Data shown are representative images of cell membrane and bound/uptaken FITC for each group $(n=4)$ (bar: $100 \mu \mathrm{m})(\mathrm{A})$ and means \pm SDs for each group $(n$ $=4$ ) of fluorescence intensity normalized against cell membrane area (B) $(* p<0.05$ vs control).

primarily via clathrin-mediated endocytosis. ${ }^{32}$ Suen et al. ${ }^{45}$ have suggested that particle size also plays an important role in the mechanism of FR internalization triggered by nanoparticle binding. Their data show that nanoparticles with hydrodynamic diameters of about 50 and $120 \mathrm{~nm}$ are internalized by both caveolae- and clathrin-mediated endocytosis pathways, whereas larger nanoparticles with a hydrodynamic diameter of around $250 \mathrm{~nm}$ are only internalized by caveolae-mediated endocytosis. ${ }^{46}$ The fact that clathrin-mediated endocytosis is observed with FITC-CNC-FA, having a hydrodynamic diameter of about $200 \mathrm{~nm}$, indicates either that the size limit for this uptake mechanism is above $200 \mathrm{~nm}$ or that the size limit depends on cell type.

Because of its large depth of field, relative to cell height, fluorescence microscopy cannot distinguish between mem-
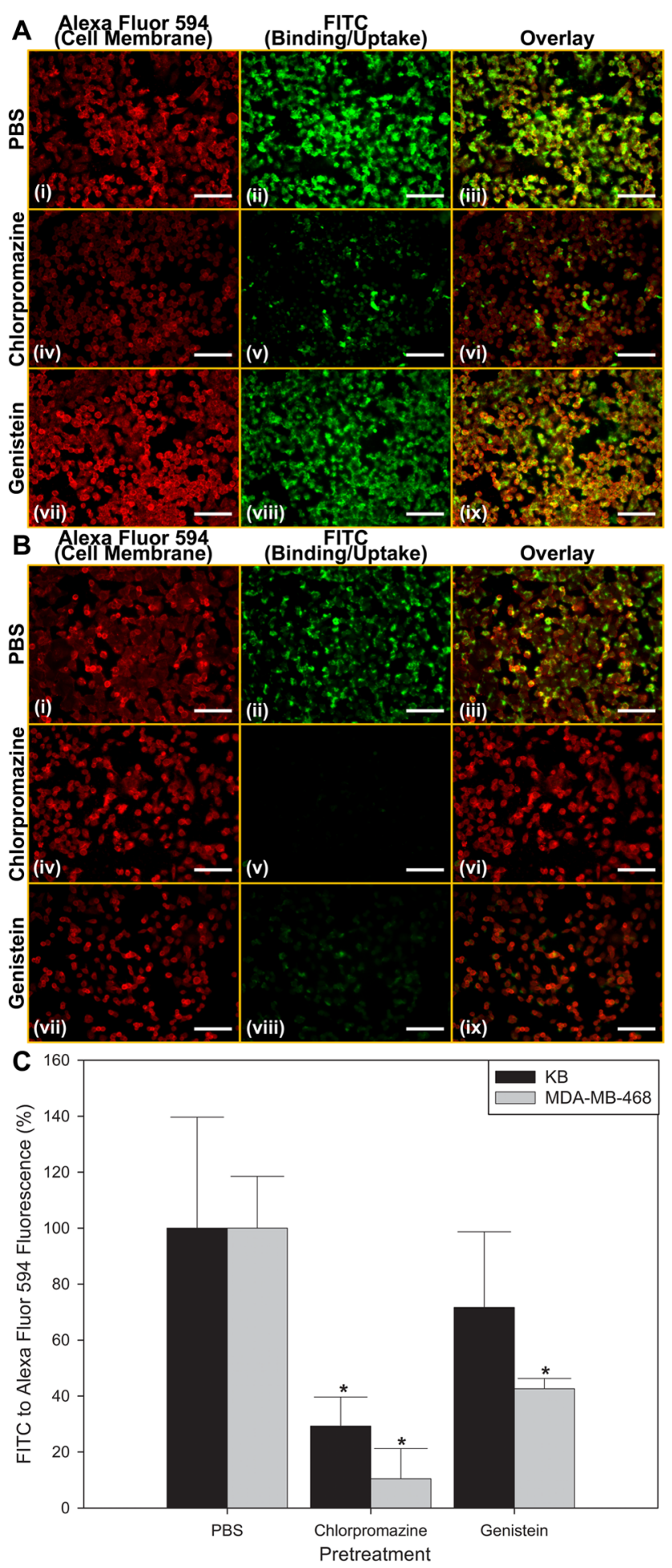

Figure 4. Effects of endocytosis inhibitors on cellular binding/uptake of FITC-CNC-FA. KB (A) and MDA-MB-648 (B) cells were pretreated with phosphate-buffered saline (PBS), $5 \mu \mathrm{g} / \mathrm{mL}$ chlorpromazine (clathrin inhibitor) or $200 \mu \mathrm{M}$ genistein (caveolae inhibitor) $30 \mathrm{~min}$, exposed to FITC-CNC-FA in the presence of the inhibitors for $2 \mathrm{~h}$, stained with Alexa Fluor 594, and imaged by fluorescence microscopy. (A) and (B) show representative images of cell membrane and bound/uptaken FITC for each group $(n=4)$ (bar: $100 \mu \mathrm{m}$ ). Data shown in (C) are means \pm SDs for each group $(n=4)$ of fluorescence intensity normalized against cell membrane area $(* p<0.05$ vs PBS). 


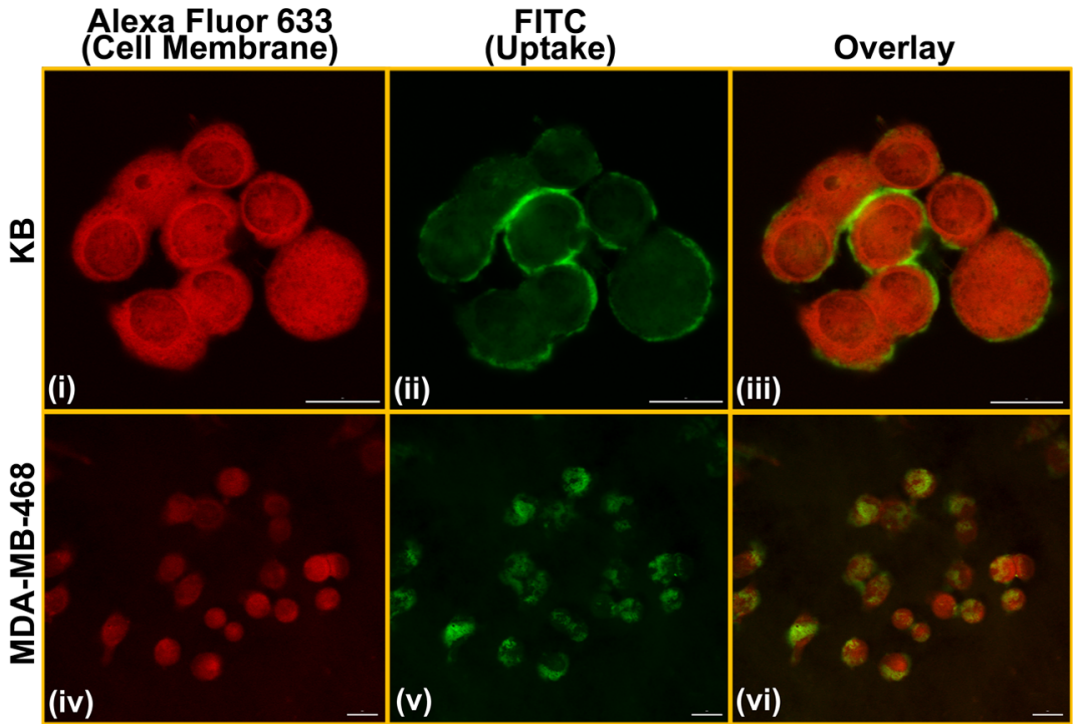

Figure 5. Cellular uptake of FITC-CNC-FA. KB (i-iii) and MDA-MB-468 (iv-vi) cells was exposed to FITC-CNC-FA for 2 h, stained with Alexa Fluor 633, and imaged by confocal microscopy. The left column of images (i, iv) show cell membrane staining, images in the middle column show FITC fluorescence, and the right column images show the overlay of the two channels. Images are representative images of cell membrane and bound and uptaken FITC for each group $(n=4)$ (bar: $20 \mu \mathrm{m})$.

brane binding and cellular uptake of nanoparticles. To assess whether FITC-CNC-FA particles are being internalized by $\mathrm{KB}$ and MDA-MB-468 cells, we recorded z-stacks of $\mathrm{KB}$ and MDA-MB-468 cells incubated in the presence of FITC-CNCFA with a confocal microscope. Cross-sectional confocal microscopy images confirmed that in addition to binding externally to the cell membrane, FITC-CNC-FA are being internalized by FR-positive cancer cells (Figure 5).

\section{CONCLUSIONS}

Advances in the biomedical field have led to the discovery of numerous nanoscale delivery vehicles. CNCs have characteristics that make them an excellent choice as a nanocarrier. We have demonstrated that once conjugated with FA, CNCs are internalized by cells via both clathrin- and caveolae-dependent, FR-mediated endocytosis pathways. We have also shown that FA-conjugated CNCs have a remarkably high affinity for the FR and thus offer highly efficient targeting of FR-positive cancer cells. The ability of FITC-CNC-FA particles to bind specifically to the membranes of FR-positive cancer cells, demonstrated in this and our previous study, ${ }^{32}$ makes them promising diagnostic bioimaging agents for the early detection of cancer. Moreover, the internalization of FITC-CNC-FA particles by FR-positive cancer cells, proven here for the first time, might enable the selective intracellular delivery of chemotherapeutic agents to such cells.

\section{EXPERIMENTAL PROCEDURES}

Materials. Dissolving-grade softwood sulfite pulp (Temalfa 93A-A) was kindly provided by Tembec, Inc. (Montréal, QC, CA). FITC, FA, genistein, doxorubicin, and dimethylsulfoxide were purchased from Sigma-Aldrich Corporation (St. Louis, MO). Chlorpromazine was purchased from MP Biomedicals (Santa Ana, CA). Folate-free RPMI 1640 medium, medium 200, low serum growth supplement, Concanavalin A Alexa Fluor 594 and 633 conjugates, and donkey anti-goat Alexa Fluor 488 were purchased from Invitrogen Corp. (Carlsbad, CA). Goat poly Immunoglobulin G (IgG) FR- $\alpha$ and goat non
IgG primary antibodies and bovine serum albumin (BSA) were purchased from Santa Cruz Biotech (Santa Cruz, CA). Antibiotics (penicillin and streptomycin) and fetal bovine serum (FBS) were purchased from Mediatech, Inc. (Manassas, VA). RNeasy Mini Kit was purchased from Qiagen (Valencia, CA). Reverse Transcription System and CellTiter-Blue Cell Viability Assay Kit were purchased from Promega (Madison, WI). TaqMan Universal PCR Master Mix, gene-specific TaqMan PCR probes and primers were purchased from Applied Biosystems (Carlsbad, CA). VECTASHIELD HardSet Mounting Media with and without 4',6-diamidino-2-phenylindole (DAPI) were purchased from Vector Labs (Burlingame, CA). Attachment factor solution (AFS) was purchased from Cell Applications (San Diego, CA). The human cancer cell lines $\mathrm{KB}$ and MDA-MB-468 were purchased from American Type Culture Collection (Manassas, VA), whereas HAEC were purchased from Invitrogen Corp. (Carlsbad, CA). All other reagents and supplies were purchased from Fisher Scientific (Pittsburgh, PA).

\section{METHODS}

CNC Preparation and CNC Conjugate Synthesis. CNCs and CNC conjugates were prepared, as described previously. ${ }^{32}$ Briefly, a CNC suspension in deionized water was prepared by hydrolysis of softwood sulfite pulp with 64 wt \% sulfuric acid at $45{ }^{\circ} \mathrm{C}$ for $1 \mathrm{~h}$ with an acid-to-pulp ratio of 10 $\mathrm{mL} / \mathrm{g}$. FITC was conjugated to the CNCs after amination of the surface hydroxyl groups with epichlorohydrin and ammonium hydroxide. FA was conjugated to the FITC-labeled CNCs (FITC-CNC) with 1-ethyl-3-(3-dimethylaminopropyl)carbodiimide and $\mathrm{N}$-hydroxysulfosuccinimide to yield FAconjugated, FITC-labeled CNCs (FITC-CNC-FA).

UV-Vis Spectroscopy. UV-vis absorption spectra were recorded using dilute suspensions of known concentration between 0.001 and $0.01 \mathrm{wt} \%$ in standard cuvettes, having an optical path length of $1 \mathrm{~cm}$, with a Thermo Scientific Evolution $300 \mathrm{UV}-$ vis spectrometer. 
$\zeta$-Potential. $\zeta$-potentials were measured in triplicate at 25 $\pm 0.1{ }^{\circ} \mathrm{C}$ in the absence of added electrolyte with a Malvern Zetasizer NanoZS particle analyzer (Malvern Instruments Ltd., Malvern, U.K.). Samples had a concentration of $0.01 \mathrm{wt} \%$ and were analyzed in Malvern DTS1060-folded capillary cells after sonication for $10 \mathrm{~min}$ in a $70 \mathrm{~W}$ Cole-Parmer 8890 ultrasonic cleaner. Reported values are mean $\zeta$-potentials obtained with the Zetasizer Nano 4.2 software using the Hückel model.

Dynamic Light Scattering (DLS). DLS measurements were conducted in triplicate at $25 \pm 0.1{ }^{\circ} \mathrm{C}$ with a Malvern Zetasizer NanoZS particle analyzer (Malvern Instruments Ltd., Malvern, U.K.). Samples had a concentration of $0.01 \mathrm{wt} \%$ and were analyzed in $12 \mathrm{~mm}$ square glass cuvettes (Malvern PCS 1115) after filtration through $0.45 \mu \mathrm{m}$ poly(vinylidenefluoride) syringe filters. Reported values are mean Peak 1 diameters obtained with the General Purpose algorithm of the Zetasizer Nano 4.2 software.

Atomic Force Microscopy (AFM). AFM images were recorded with an Asylum Research MFP-3D Bio atomic force microscope (Asylum Research, Santa Barbara, CA). Aqueous suspensions were diluted to $0.001 \mathrm{wt} \%$ and sonicated at 150 $\mathrm{W}$ for $3 \mathrm{~min}$. Single drops of the suspensions were placed on freshly cleaved mica surfaces and allowed to dry under ambient conditions. Samples were scanned in intermittent contact mode under ambient conditions with Olympus AC160TS tips (nominal tip radius $<10 \mathrm{~nm}$, spring constant $42 \mathrm{~N} / \mathrm{m}$ ).

Cell Culture. HAEC were grown in Medium 200 supplemented with low serum growth supplement. $\mathrm{KB}$ and MDA-MB-468 cells were grown in folate-free RPMI 1640 medium supplemented with $10 \%$ FBS. Cells were grown at 37 ${ }^{\circ} \mathrm{C}$ and $5 \% \mathrm{CO}_{2}$. All experiments were conducted in basal medium.

Real-Time Reverse Transcription-Polymerase Chain Reaction (RT-PCR). Total RNA from HAEC, KB, and MDAMB-468 cells was isolated and purified using RNeasy Mini Kit according to the protocol of the manufacturer. Quantitative real-time RT-PCR using TaqMan probes and primers were used for gene expression analyses, as described previously. ${ }^{47}$ Amplification of individual genes was performed with Applied Biosystems 7300 real-time PCR system using TaqMan Universal PCR Master Mix and a standard thermal cycler protocol. TaqMan Gene Expression Assay Reagents for human FR- $\alpha$ and glyceraldehyde 3-phosphate dehydrogenase were used for specific probes and primers of PCR amplifications. The threshold cycle $\left(C_{T}\right)$ was determined, and relative quantification was calculated by the comparative $C_{T}$ method as described previously. ${ }^{47}$ PCR results were observed and analyzed using a 7300 Real-time PCR System and 7300 Realtime PCR System Sequence Detection Software v1.2.3 (Applied Biosystems, Carlsbad, CA).

Fluorescence Microscopy. HAEC, KB, and MDA-MB468 cells were seeded onto Lab-Tek Chamber Slide systems coated with AFS and grown to confluency. Before imaging, cells were washed and mounted with VECTASHIELD HardSet Mounting Medium with or without DAPI. Images were obtained with a Leica AF6000 fluorescent microscope (Leica Microsystems Inc., Buffalo Grove, IL). A quantitative comparison of experimental groups was conducted by measuring the total fluorescence intensity normalized by cell area using ImageJ software (National Institutes of Health, Bethesda, MD).

Immunofluorescent Staining. Cells were washed and fixed with $4 \%$ paraformaldehyde for $15 \mathrm{~min}$. Cells were again washed and blocked with $3 \%$ BSA for $1 \mathrm{~h}$. Cells were then treated with $4 \mu \mathrm{g} / \mathrm{mL}$ goat poly IgG FR- $\alpha$ primary antibody or goat non IgG antibody in $1 \%$ BSA solution overnight at $4{ }^{\circ} \mathrm{C}$. Cells were thoroughly washed and treated with $2 \mu \mathrm{g} / \mathrm{mL}$ donkey anti-goat Alexa Fluor 488 in 1\% BSA solution for $2 \mathrm{~h}$ at room temperature.

Binding/Uptake Assay. Cells were incubated with FITC, FITC-CNC, or FITC-CNC-FA at a FITC concentration of 5 $\mu \mathrm{g} / \mathrm{mL}$ for $2 \mathrm{~h}$, corresponding to FITC-CNC and FITC-CNCFA concentrations of 67.6 and $135.1 \mu \mathrm{g} / \mathrm{mL}$, respectively. Cells were washed twice with phosphate-buffered saline (PBS), and the plasma membranes were briefly stained with $50 \mathrm{ng} / \mu \mathrm{L}$ of Concanavalin A Alexa Fluor 594 for $2 \mathrm{~min}$. Cells were then fixed with cold ethanol for $1 \mathrm{~h}$.

Free Folate Inhibition Assay. Cells were incubated with FITC-CNC-FA at a FITC concentration of $5 \mu \mathrm{g} / \mathrm{mL}$ in the presence of $0,5,10$, or $25 \mathrm{mM}$ FA for $2 \mathrm{~h}$. Cells were washed twice with PBS, and the plasma membranes were briefly stained with $50 \mathrm{ng} / \mu \mathrm{L}$ of Concanavalin A Alexa Fluor 594 for $2 \mathrm{~min}$. Cells were then fixed with cold ethanol for $1 \mathrm{~h}$.

Binding/Uptake Mechanism Assay. Cells were pretreated with PBS, $5 \mu \mathrm{g} / \mathrm{mL}$ chlorpromazine, or $200 \mu \mathrm{M}$ genistein for $30 \mathrm{~min}$. Growth medium was replaced with basal medium containing FITC-CNC-FA at a FITC concentration of $5 \mu \mathrm{g} / \mathrm{mL}$ and either PBS, $5 \mu \mathrm{g} / \mathrm{mL}$ chlorpromazine (clathrin inhibitor), or $200 \mu \mathrm{M}$ genistein (caveolae inhibitor). After $2 \mathrm{~h}$ of exposure, cells were washed twice with PBS, and the plasma membranes were briefly stained with $50 \mathrm{ng} / \mu \mathrm{L}$ of Concanavalin A Alexa Fluor 594 for 2 min. Cells were then fixed with cold ethanol for $1 \mathrm{~h}$.

Confocal Microscopy. KB and MDA-MB-468 cells were seeded onto round coverslips coated with AFS and allowed to grow for $24 \mathrm{~h}$ at $37{ }^{\circ} \mathrm{C}$. Cells were incubated with FITC, FITC-CNC, or FITC-CNC-FA at a FITC concentration of 5 $\mu \mathrm{g} / \mathrm{mL}$ for $2 \mathrm{~h}$. Cells were washed twice with PBS, and the plasma membranes were briefly stained with $50 \mathrm{ng} / \mu \mathrm{L}$ of Concanavalin A Alexa Fluor 633 for $2 \mathrm{~min}$. Cells were then fixed with cold ethanol for $1 \mathrm{~h}$, washed twice with PBS, and mounted with VECTASHIELD HardSet Mounting Medium. Images were obtained using a Zeiss LSM 510 laser scanning microscope equipped with argon and helium-neon lasers (Carl Zeiss Microscopy, LLC, Thornwood, NY). Images were analyzed using ZEN 2011 blue edition software (Carl Zeiss, Oberkochen, Germany).

Statistical Analysis. All statistical analysis of data was performed using SigmaPlot 11 (SPSS Inc., Chicago, IL). Oneway analysis of variance was used to compare mean responses among the treatments. For each endpoint, the treatment means were compared using the Holm-Sidak method. Statistical probability of $p<0.05$ was considered significant.

\section{ASSOCIATED CONTENT}

\section{Supporting Information}

The Supporting Information is available free of charge on the ACS Publications website at DOI: 10.1021/acsomega.8b01619.

FR- $\alpha$ expression in HAEC KB, and MDA-MB-468 cells; preliminary in vivo targeting data in a mouse model; tumor sizes over the course of in vivo study; mouse weights over the course of the in vivo study (PDF) 


\section{AUTHOR INFORMATION}

\section{Corresponding Author}

*E-mail: maren.roman@vt.edu. Phone: (540) 231-1421. Fax: (540) 231-8176.

\section{ORCID}

Maren Roman: 0000-0001-6622-8591

\section{Notes}

The authors declare no competing financial interest.

\section{ACKNOWLEDGMENTS}

This material is based upon work supported by the National Research Initiative of the USDA Cooperative State Research, Education and Extension Service under Grant 2005-3550416088 and the National Science Foundation under Grants CHE-0724126 and DMR-0907567. Partial fundings from the Institute for Critical Technology and Applied Science, the Macromolecules Innovation Institute, and Omnova Solutions, Inc., are also acknowledged. The authors furthermore thank Tembec, Inc. for the pulp sample.

\section{ABBREVIATIONS}

AFM, atomic force microscopy; AFS, attachment factor solution; BSA, bovine serum albumin; CNCs, cellulose nanocrystals; DAPI, 4',6-diamidino-2-phenylindole; DLS, dynamic light scattering; FA, folic acid; FBS, fetal bovine serum; FITC, fluorescein-5'-isothiocyanate; FR, folate receptor; IgG, Immunoglobulin G; HAEC, human aortic endothelial cells; PBS, phosphate-buffered saline; PCR, polymerase chain reaction; RT-PCR, reverse transcription-polymerase chain reaction

\section{REFERENCES}

(1) Raffa, V.; Riggio, C.; Smith, M. W.; Jordan, K. C.; Cao, W.; Cuschieri, A. BNNT-Mediated Irreversible Electroporation: Its Potential on Cancer Cells. Technol. Cancer Res. Treat. 2012, 11, 459-65.

(2) Kona, S.; Dong, J. F.; Liu, Y. L.; Tan, J. F.; Nguyen, K. T. Biodegradable nanoparticles mimicking platelet binding as a targeted and controlled drug delivery system. Int. J. Pharm. 2012, 423, 516524.

(3) National Cancer Institute, Cancer Treatment: Targeted Cancer Therapies. https://www.cancer.gov/about-cancer/treatment/types/ targeted-therapies/targeted-therapies-fact-sheet?redirect $=$ true (accessed Oct. 23, 2018).

(4) Dong, S.; Hirani, A.; Colacino, K. R.; Lee, Y. W.; Roman, M. Cytotoxicity and Cellular Uptake of Cellulose Nanocrystals. Nano LIFE 2012, 2, No. 1241006.

(5) Bai, M.; Bornhop, D. J. Recent Advances in Receptor-Targeted Fluorescent Probes for In Vivo Cancer Imaging. Curr. Med. Chem. 2012, 19, 4742-4758.

(6) Decuzzi, P.; Godin, B.; Tanaka, T.; Lee, S. Y.; Chiappini, C.; Liu, X.; Ferrari, M. Size and shape effects in the biodistribution of intravascularly injected particles. J. Controlled Release 2010, 141, 3207.

(7) Godin, B.; Driessen, W. H.; Proneth, B.; Lee, S. Y.; Srinivasan, S.; Rumbaut, R.; Arap, W.; Pasqualini, R.; Ferrari, M.; Decuzzi, P. An integrated approach for the rational design of nanovectors for biomedical imaging and therapy. Adv. Genet. 2010, 69, 31-64.

(8) Habibi, Y.; Lucia, L. A.; Rojas, O. J. Cellulose nanocrystals: chemistry, self-assembly, and applications. Chem. Rev. 2010, 110, 3479-500.

(9) Owens, D. E., III; Peppas, N. A. Opsonization, biodistribution, and pharmacokinetics of polymeric nanoparticles. Int. J. Pharm. 2006, 307, 93-102.
(10) Lacerda, L.; Herrero, M. A.; Venner, K.; Bianco, A.; Prato, M.; Kostarelos, K. Carbon-Nanotube Shape and Individualization Critical for Renal Excretion. Small 2008, 4, 1130-1132.

(11) Boso, D. P.; Lee, S. Y.; Ferrari, M.; Schrefler, B. A.; Decuzzi, P. Optimizing particle size for targeting diseased microvasculature: from experiments to artificial neural networks. Int. J. Nanomed. 2011, 6, $1517-26$

(12) Gaur, P. K.; Mishra, S.; Gupta, V. B.; Rathod, M. S.; Purohit, S.; Savla, B. A. Targeted drug delivery of Rifampicin to the lungs: formulation, characterization, and stability studies of preformed aerosolized liposome and in situ formed aerosolized liposome. Drug Dev. Ind. Pharm. 2010, 36, 638-646.

(13) Kamps, J. A. A. M.; Asgeirsdottir, S. A.; Morselt, H. W. M.; Bartsch, M.; Scherphof, G. L.; Molema, G. Targeted liposome mediated drug delivery into endothelial subsets and endothielial cells in inflammatory sites. J. Vasc. Res. 2006, 43, 10. (Suppl.1)

(14) Wang, H.; Zhao, P. Q.; Su, W. Y.; Wang, S.; Liao, Z. Y.; Niu, R. F.; Chang, J. PLGA/polymeric liposome for targeted drug and gene co-delivery. Biomaterials 2010, 31, 8741-8748.

(15) Asadishad, B.; Vosoughi, M.; Alamzadeh, I.; Tavakoli, A. Synthesis of Folate-Modified, Polyethylene Glycol-Functionalized Gold Nanoparticles for Targeted Drug Delivery. J. Dispersion Sci. Technol. 2010, 31, 492-500.

(16) Wang, Y.; Newell, B. B.; Irudayaraj, J. Folic Acid Protected Silver Nanocarriers for Targeted Drug Delivery. J. Biomed. Nanotechnol. 2012, 8, 751-759.

(17) Cao, W.; Zhou, J.; Mann, A.; Wang, Y.; Zhu, L. FolateFunctionalized Unimolecular Micelles Based on a Degradable Amphiphilic Dendrimer-Like Star Polymer for Cancer Cell-Targeted Drug Delivery. Biomacromolecules 2011, 12, 2697-2707.

(18) Sideratou, Z.; Kontoyianni, C.; Drossopoulou, G. I.; Paleos, C. M. Synthesis of a folate functionalized PEGylated poly(propylene imine) dendrimer as prospective targeted drug delivery system. Bioorg. Med. Chem. Lett. 2010, 20, 6513-6517.

(19) Fleming, K.; Gray, D. G.; Matthews, S. Cellulose crystallites. Chemistry 2001, 7, 1831-5.

(20) de Souza Lima, M. M.; Borsali, R. Rodlike cellulose microcrystals: Structure, properties, and applications. Macromol. Rapid Commun. 2004, 25, 771-787.

(21) Roman, M. Toxicity of Cellulose Nanocrystals: A Review. Ind. Biotechnol. 2015, 11, 25-33.

(22) Dainty, L. A.; Risinger, J. I.; Morrison, C.; Chandramouli, G. V.; Bidus, M. A.; Zahn, C.; Rose, G. S.; Fowler, J.; Berchuck, A.; Maxwell, G. L. Overexpression of folate binding protein and mesothelin are associated with uterine serous carcinoma. Gynecol. Oncol. 2007, 105, $563-70$.

(23) Hartmann, L. C.; Keeney, G. L.; Lingle, W. L.; Christianson, T. J. H.; Varghese, B.; Hillman, D.; Oberg, A. L.; Low, P. S. Folate receptor overexpression is associated with poor outcome in breast cancer. Int. J. Cancer 2007, 121, 938-942.

(24) Toffoli, G.; Cernigoi, C.; Russo, A.; Gallo, A.; Bagnoli, M.; Boiocchi, M. Overexpression of folate binding protein in ovarian cancers. Int. J. Cancer 1997, 74, 193-198.

(25) Weitman, S. D.; Frazier, K. M.; Kamen, B. A. The folate receptor in central nervous system malignancies of childhood. $J$. Neurooncol. 1994, 21, 107-112.

(26) Weitman, S. D.; Weinberg, A. G.; Coney, L. R.; Zurawski, V. R.; Jennings, D. S.; Kamen, B. A. Cellular localization of the folate receptor: potential role in drug toxicity and folate homeostasis. Cancer Res. 1992, 52, 6708-11.

(27) Weitman, S. D.; Lark, R. H.; Coney, L. R.; Fort, D. W.; Frasca, V.; Zurawski, V. R., Jr.; Kamen, B. A. Distribution of the folate receptor GP38 in normal and malignant cell lines and tissues. Cancer Res. 1992, 52, 3396-3401.

(28) Hutson, J. R.; Stade, B.; Lehotay, D. C.; Collier, C. P.; Kapur, B. M. Folic Acid Transport to the Human Fetus Is Decreased in Pregnancies with Chronic Alcohol Exposure. PLoS One 2012, 7, No. e38057. 
(29) Salazar, M. D.; Ratnam, M. The folate receptor: what does it promise in tissue-targeted therapeutics? Cancer Metastasis Rev. 2007, $26,141-52$.

(30) Society, A. C. Cancer Facts \& Figures 2014; American Cancer Society: Atlanta, GA, 2014.

(31) Society, A. C. Cancer Prevention \& Early Detection Facts \& Figures; American Cancer Society: Atlanta, GA, 2014.

(32) Dong, S.; Cho, H. J.; Lee, Y. W.; Roman, M. Synthesis and cellular uptake of folic acid-conjugated cellulose nanocrystals for cancer targeting. Biomacromolecules 2014, 15, 1560-1567.

(33) Cole, L.; Coleman, J.; Evans, D.; Hawes, C. Internalisation of fluorescein isothiocyanate and fluorescein isothiocyanatedextran by suspension-cultured plant cells. J. Cell Sci. 1990, 96, 721-730.

(34) Wang, S.; Lee, R. J.; Mathias, C. J.; Green, M. A.; Low, P. S. Synthesis, purification, and tumor cell uptake of $67 \mathrm{Ga}$-deferoxaminefolate, a potential radiopharmaceutical for tumor imaging. Bioconjug. Chem. 1996, 7, 56-62.

(35) Mathias, C. J.; Wang, S.; Lee, R. J.; Waters, D. J.; Low, P. S.; Green, M. A. Tumor-selective radiopharmaceutical targeting via receptor-mediated endocytosis of gallium-67-deferoxamine-folate. J. Nucl. Med. 1996, 37, 1003-1008.

(36) Sudimack, J.; Lee, R. J. Targeted drug delivery via the folate receptor. Adv. Drug Delivery Rev. 2000, 41, 147-162.

(37) Quintana, A.; Raczka, E.; Piehler, L.; Lee, I.; Myc, A.; Majoros, I.; Patri, A. K.; Thomas, T.; Mule, J.; Baker, J. R., Jr. Design and function of a dendrimer-based therapeutic nanodevice targeted to tumor cells through the folate receptor. Pharm. Res. 2002, 19, 13101316.

(38) Destito, G.; Yeh, R.; Rae, C. S.; Finn, M. G.; Manchester, M. Folic acid-mediated targeting of cowpea mosaic virus particles to tumor cells. Chem. Biol. 2007, 14, 1152-1162.

(39) Nukolova, N. V.; Oberoi, H. S.; Cohen, S. M.; Kabanov, A. V.; Bronich, T. K. Folate-decorated nanogels for targeted therapy of ovarian cancer. Biomaterials 2011, 32, 5417-5426.

(40) Leamon, C. P.; DePrince, R. B.; Hendren, R. W. Folatemediated drug delivery: effect of alternative conjugation chemistry. $J$. Drug Target. 1999, 7, 157-169.

(41) Gabrielson, N. P.; Pack, D. W. Efficient polyethyleniminemediated gene delivery proceeds via a caveolar pathway in HeLa cells. J. Controlled Release 2009, 136, 54-61.

(42) Chen, L.; Xiong, S.; She, H.; Lin, S. W.; Wang, J.; Tsukamoto, $\mathrm{H}$. Iron causes interactions of TAK1, p21ras, and phosphatidylinositol 3-kinase in caveolae to activate IkappaB kinase in hepatic macrophages. J. Biol. Chem. 2007, 282, 5582-5588.

(43) Gumbleton, M.; Abulrob, A. G.; Campbell, L. Caveolae: an alternative membrane transport compartment. Pharm. Res. 2000, 17, 1035-1048.

(44) Rejman, J.; Bragonzi, A.; Conese, M. Role of clathrin- and caveolae-mediated endocytosis in gene transfer mediated by lipo- and polyplexes. Mol. Ther. 2005, 12, 468-474.

(45) Suen, W. L. L.; Chau, Y. Size-dependent internalisation of folate-decorated nanoparticles via the pathways of clathrin and caveolae-mediated endocytosis in ARPE-19 cells. J. Pharm. Pharmacol. 2014, 66, 564-573.

(46) Suen, W. L.; Chau, Y. Specific uptake of folate-decorated triamcinolone-encapsulating nanoparticles by retinal pigment epithelium cells enhances and prolongs antiangiogenic activity. $J$. Controlled Release 2013, 167, 21-28.

(47) Lee, W. H.; Sonntag, W. E.; Mitschelen, M.; Yan, H.; Lee, Y. W. Irradiation induces regionally specific alterations in pro-inflammatory environments in rat brain. Int. J. Radiat. Biol. 2010, 86, 132-44. 